\title{
Rapid review of AGPs published
}

By Manas Dave

\section{The COVID-19 Dental Services Evidence Review (CoDER)}

Working Group, led by Professors Jan Clarkson and Craig Ramsay, have conducted a rapid review to assess dental aerosol generating procedures (AGPs) and their mitigation in international guidance documents. This has been published in Cochrane Oral Health and aims to support decision-makers and a review being undertaken by the Scottish Dental Clinical Effectiveness Programme (SDCEP). ${ }^{1}$ Both English and Spanish translations are available for the summary infographic with a French translation available soon.

The aim of this rapid review was to assess how dental AGPs were defined in international dental guidelines, the reported recommended time gaps between patients in surgery following an AGP (fallow periods) and mitigation processes advised with personal protective equipment (PPE), dental procedures and the environment in both COVID-19 and non-COVID-19 patients. The appendices provide details of the individual guidance documents included and their reports on the aforementioned factors.

The working group have further objectives to assess the relationship between COVID-19 epidemiology and World Bank income status with PPE provision and the quality of the linked evidence to recommendations. These are currently in progress and aimed to be reported later.

\section{Brief summary of selected key messages}

This rapid review initially identified 74 guidance documents from 72 countries; following exclusions, 63 documents from 58 countries were included. Ninety-eight percent of countries stated that AGPs could be provided for non-COVID-19 patients, however, only $56 \%$ of documents provided a definition of AGPs. Regarding PPE, 33\% of countries recommended surgical masks for non-COVID-19 patients while $70 \%$ and $19 \%$ recommended the use of FFP2/N95 masks and FFP3 masks respectively. A fallow period after undertaking AGPs in non-COVID-19 patients was recommended in $48 \%$ of documents, however, ranged from two to 180 minutes. Notably, there is limited evidence to support the majority of recommendations in the guidance documents.

An infographic illustration is available at: https://bit.ly/2DeiLwX.

\section{Reference}

1. Clarkson J, Ramsay C, Richards D, Robertson C, Aceves-Martins M; on behalf of the CoDER Working Group (2020). Aerosol Generating Procedures and their Mitigation in International Dental Guidance Documents - A Rapid Review. Cochrane Oral Health, 24 July 2020. Available at: https://oralhealth.cochrane. org/news/aerosol-generating-procedures-and-their-mitigation-internationalguidance-documents (accessed July 2020). 